\title{
Empowering Artificial Intelligence and Cyber SecurityChallenges in Smart Manufacturing
}

\section{Dr.P.KALARANI}

Assistant Professor, Department of Computer Technology and Information Technology

Kongu Arts and Science College, Erode, Tamilnadu, India

Mail id:meet.kalaram@gmail.com

Article History:Received:11 november 2020; Accepted: 27 December 2020; Published online: 05 April 2021 Abstract:SM (Smart Manufacturing) is a broad category of manufacturing that employs a computer-based
integrated manufacturing system, with a higher end of new adaptability and quick change in design structure,
along with digitalization and effective workforce training. It is necessary to incorporate new techniques in the
SM system to adapt to the changes in the present system. Smart factories increase the output of the unit, quality,
andconsistency maintenance by satisfaction for the customers. Smarter technology helps to get information with
the help of computed technology in the organization through which the information/data will be recorded
periodically. The Smart Manufacturing systemwhich is very safer for the environment is known to us as Green
Manufacturing (GM). Green-tech or Green Manufacturing is an umbrella term which comes under the same
branch in one way or another which is used in several technologies or the field of science in order to bring up
with products which are eco-friendly for the environment. GM is the most needed one which may lead to a
higher level of development in the aspect of the economy. Moreover, the confidentiality of the information and
the vulnerabilities they come with SM systems is also needs to be solved when it comes to Cyber security.
Hence, we have proposed an efficient green manufacturing approach in SM systems with the aid of Artificial
Intelligence (AI) and cyber security frameworks. The proposed work employs a Dual stage ANN to find the
design configuration of SM systems in industries. Then, for maintaining the data confidentiality while
communication, the data are encrypted by using 3DES approach.

Keywords: Smart manufacturing, Artificial Intelligence, Cyber Security, Confidentiality, Encryption.

\section{Introduction}

The advanced technological revolution and revolution in industries are gaining more attention. The new era is getting enhanced with the usage of the internet along with the integration of AI in it which is responsible for bringing in more changes in the upcoming time in several fields. The other technologies rapped up with the internet and AI in their application will bring advancement in that particular field and will definitely be a game-changer of that corresponding place. When compared to other industries manufacturing sectors are capable of providing a livelihood for many. The fusion of several technologies like communication, intelligent-based, product-based technology in manufacturing industries is considered as a game-changer in that sector in terms of a newapproach in manufacturing and its entire system.

*Corresponding author: Dr.P.KALARANI

Assistant Professor, Department of Computer Technology and Information Technology

Kongu Arts and Science College, Erode, Tamilnadu, India

Mail id:meet.kalaram@gmail.com 


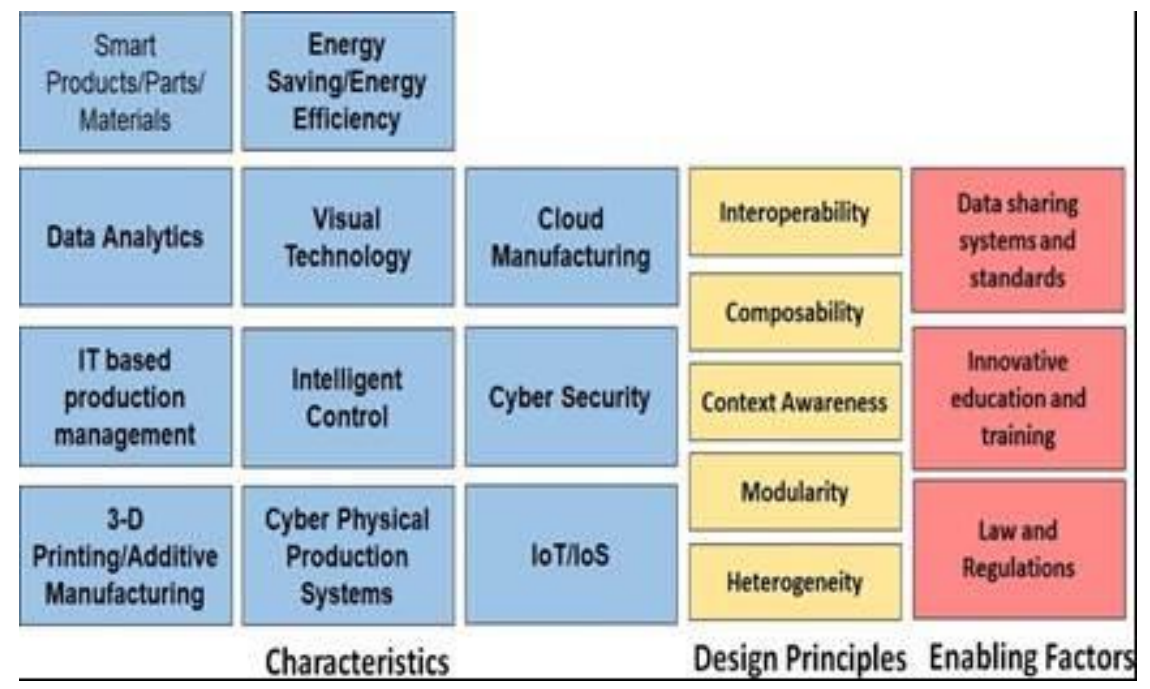

Fig. 1. Characteristics, design principles and enabling technology defining smartmanufacturing

\section{New Development of Artificial Intelligence}

AI has proved its advancement in several applications like sensors, big data management, e-commerce, development of information technology, space, cybersecurity, the structure and pattern of AI have brought in advancement in its system itself known as Artificial Intelligence 2.0. AI 2.0 is the new stage of artificial intelligence research which is highly different from that of the past 60 years older one. The newer version of AI known as the AI 2.0 helps in intensive deep learning (DL) with the obtained data, swarm intelligence, hybrid-based augmented reality, cross-media engagement, etc. The evolution in Smart city, medical field, transportation, logistics, robotics, smart vehicles, smartphones, toys, etc. with the help of artificial intelligence creates a huge demand in market space which drives the value and need of the products more than before.

\section{Artificial Intelligence Facilitates for Intelligent Manufacturing}

Intelligent manufacturing is considered as the new manufacturing technique which has given scope for development in information technology, communication, advanced science, manufacturing sector, engineering, product manufacturing technology, which arealso integrated by the means of the entire network with a new development from the base level.

Intelligent manufacturing is a wider range of innovation in manufacturing with the purpose of increased production, and transaction of products with the aim of utilizing advanced information in the manufacturing sector. The benefit of adapting intelligent manufacturing helps in detecting the activity and response to information, which same time helps in increasing the yield, quality, reduction in downtime, and also leads to improved overall equipment effectiveness. Through which competitive structure in the market improves by which particular industries with intelligent manufacturing will be able to show its capability as a stronger competitor in that firm among other competitors.

$\mathrm{Li}$ et al, AI technology has brought in development in the model in all aspects from the basic level of the system up to the end of the system. This new adaption of techniques in the system helps in providing better production and a better service system to the users.

The new means of adaption in technology involves smart manufacturing with digitalization, IoT, virtual reality, a collaboration of technology, flexibility, and intelligent-system. The new forms of adaption in technology involve intelligent manufacturing with interconnection, data-driving, cross-sector integration, innovation, and an autonomy-based intelligent system. The deep integration in this new model is about the usage of all new means, and form in the advanced intelligent system. Fig 2 illustrates this structure in detail as follows. 


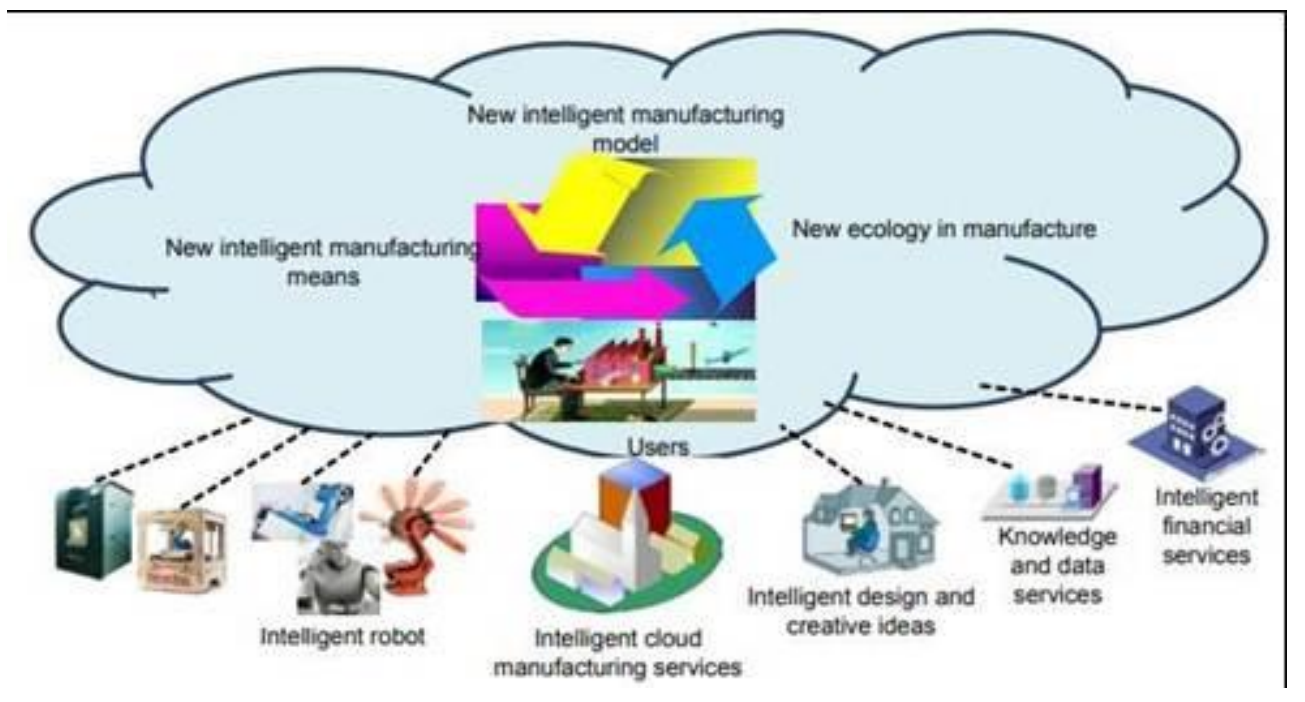

Fig. 2 New models, means, and forms of intelligent manufacturing

\section{Current Status and Problems of Green Manufacturing \\ 4.1 Green Manufacturing}

It is a well-known fact that the manufacturing industry has faced 3rd major revolutions in this sector and ongoing is the 4th revolution which is illustrated in fig 3 . The first three revolutions helped in bringing change in the production and development of the economy. The present 4 th revolution is described as SMART MANUFACTURING which is expected to bring in changes with the help of utilizing a more advanced technological system.

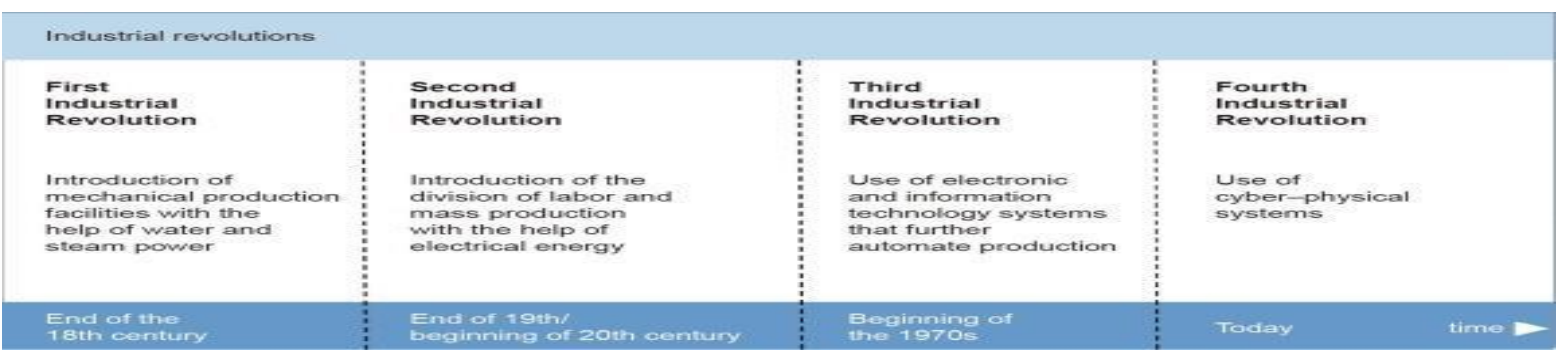

Fig. 3. Four industrial revolutions.

[13] Chemicals and petroleum were considered as an important component for the process industry. The petrochemical industry has brought a big change for the development of the economy of the country but it has brought an equal amount of negative aspects in health and environmental damage for people by the means of a frequent accident and raise in pollution level.

[14] In this present era accidents in industries gain more attention because of widespread information through news, and social media. This makes the government more careful by providing a standard set of rules and regulations for industries/factories. This creates more demand for Green Manufacturing.

[16] The cause of environmental damage because of the petrochemical industry will have more impact on the surrounding system. When considering the demand for the process industry that is gained in India, there is an immediate need for addressing the issue of energy consumption and emission by that industry need for immediate environmental protection.

$[17,18]$ Green Manufacturing is the only solution to achieve safety, lesserconsumption of energy, and lesserlevel of emission from industries. It tries to track the issue with the help of process life-cycle through smart monitoring, beforehand warning, effective decision making, and pollution reduction. It can improve the efficiency and safety of process industries, and it is highly demanded the means of economic development.

\subsection{Current Status and Major Problems}

The problems faced by industries due to green manufacturing has been discussed as follows in the sections below.

Isolation of Information among Multiple Fields 
In the process industry, some factors like storage, production, and transportation, waste management are interrelated to one and another. But each factor depends on their level of information and data. The exchange of inappropriate information to the system may lead to improper functioning of the life-cycle of the system. For example, if misleading or late information about hazardous chemical transportation will put the entireprocess at risk if information delivered at the right time would have saved from accidents and things that must be done at the right time will be performed. So information must be transferred at the right time lack of information passing may lead to a serious issue. In order to overcome this, a better information passing system must be adopted in the system to implement green manufacturing in industries.

\section{Diverse information types and different kinds of data}

The different levels of knowledge will be preferred for the different processes such as transportation, manufacturing, and storage have their own level of information system and working system. The information about pressure, temperature level is important to overcome the unexpected situation and other issues. In the aspect of transportation, the information about the destination, status of a vehicle, information about drivers is considered to be important. The above-mentioned information will sometime be stored in a different system in such a situation it will be difficult to process the information to one and another due to format in data, and information collecting method. When proper information is not obtained at right time may lead to a lack of a process of the life-cycle of the system. There are different methods involved during the process of collecting information and computing all those collected information in the system may requiremore time and be considered a challenging task.

\section{Lack of a Process-Safety-Oriented Decision-Making System}

The utilization of Geometric magnification in the process industry and petrochemical industry will help in reducing the cost and helps in gaining more benefitsin the firm. Which enables larger production and supply in the market space. The industry will be seen as one within a single city, individual province, but the result of support will be seen as a whole across the nation. Industries in the aspect of production and supply need help national-wide for the purpose of transport, storing materials, in order to satisfy the needs of the customer who live in areas populated in a different region. The bulk storage of hazardous material may lead to risk in storage for nearby communities.

In production, the scale-up process needs full control of the system, perfect operation by a human, and good condition mechanical integrity. If problems are not handled properly in the initial stage may lead to a bigger error in the system in the future. Rick management technique has been taken into consideration while developing petrochemical plants with the support of tackling the problem during dangerous situation by identifying and managing it some risk management analysis may help in a typical situation they are HAZOP (hazard and operability analysis), LOPA (layer of protection analysis), SIL (system integrity level).

The problem must be analysed from the base level so that the error from the beginning stage will be eradicated but maintaining risk management information is considered a challenging task. [19,20] the operator behavior is highly dependent onprovided training and management skills to him. The accident which occurred in a chemical factory in Germany known as BASF leads to economic loss and casualties. Thisaccident was due to the operation failure of one part of the tools/machine. A proper decision must be taken before operating a system in order to overcome operation failure. In such a situation an intelligent system is needed to handle the issue and suggest proper steps to overcome it.

This kind of system will help with the progress of the system when compared to human capacity. One of the important factors for practicing safety is to have a system with no error or mistake so that hazardous situation will never occur in real-time.

\section{Lack of Early Warning and Risk Tracing Systems}

[21] The accidents in the process industry are very severe due to lack of an efficient warning. The operation petrochemical industry is based on its own level of restriction in each sector and they are capable of addressing it. But the small effect will sometimes lead to a bigger instance and the system will face downstream because of critical issues.

The accidents will be addressed with the experience of dealing with the same kind of instance which would have happened before. To overcome these problems the system must be capable of understanding the abnormalities as soon as possible so that theproblem will be addressed in the initial stage itself. The diagnosing function must provide a solution for the present real-time problem and must have the potential for handling it and to overturn the situation to the normal one. A decision-making system is needed to deal with the issue. But due to several reasons, a smart system for dealing with accidental situations is still not available at the present time. 


\section{Artificial Intelligence for Green Manufacturing}

[22] Machine Intelligence is the branch of automation and CS(Computer Science). With Artificial intelligence, machine intelligence will interact with machines or the environment intelligently. Artificial intelligence combines with other branches like CS, Engineering, mathematics, automation, and psychology to obtain

knowledge from that branch. The issues/problems faced by artificial intelligence has beenillustrated in fig 4 as follows.

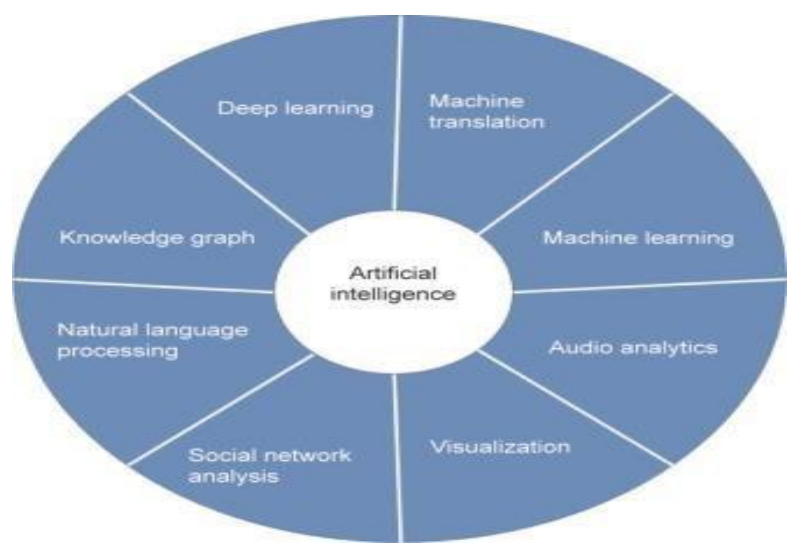

Fig. 4. Several sub-problems in artificial intelligence.

The problems of Green Manufacturing can be categorized into 4 groups based on theircharacteristics:

- Integration of information

- Risk management

- Help during decision making

- Early warming

Many techniques and solutions can address the problems faced by Green Manufacturing some of the problemsolving factors will be discussed in detail in the following section.

\subsection{Information integration via knowledge graphs}

Knowledge Graph is the best-known technique involved in addressing linked data management in Artificial intelligence. This graph is a sematic structure that helps in describing the concept and the relationship within the system. This graph will provide reasoning and advanced rules based on the concept of deep learning (DL). [23,24]knowledge Graphs are highly preferred in internet-based applications like social networking, internet-based financial system, social security, and encyclopedia.

The process industry needs more specific information in the areas of specialization in the fields of chemical engineering, safety, control, automation system, and mechanical side. The knowledge graph can only be implemented in the industry with a complete idea about the working structure of the graph. [25] Implementation of the knowledge graph needs more understanding of the required field. Fig 4 illustrates the model for building a knowledge graph the implementation of the knowledge graph is categorized into 3 stages are discussed in the sections below. 


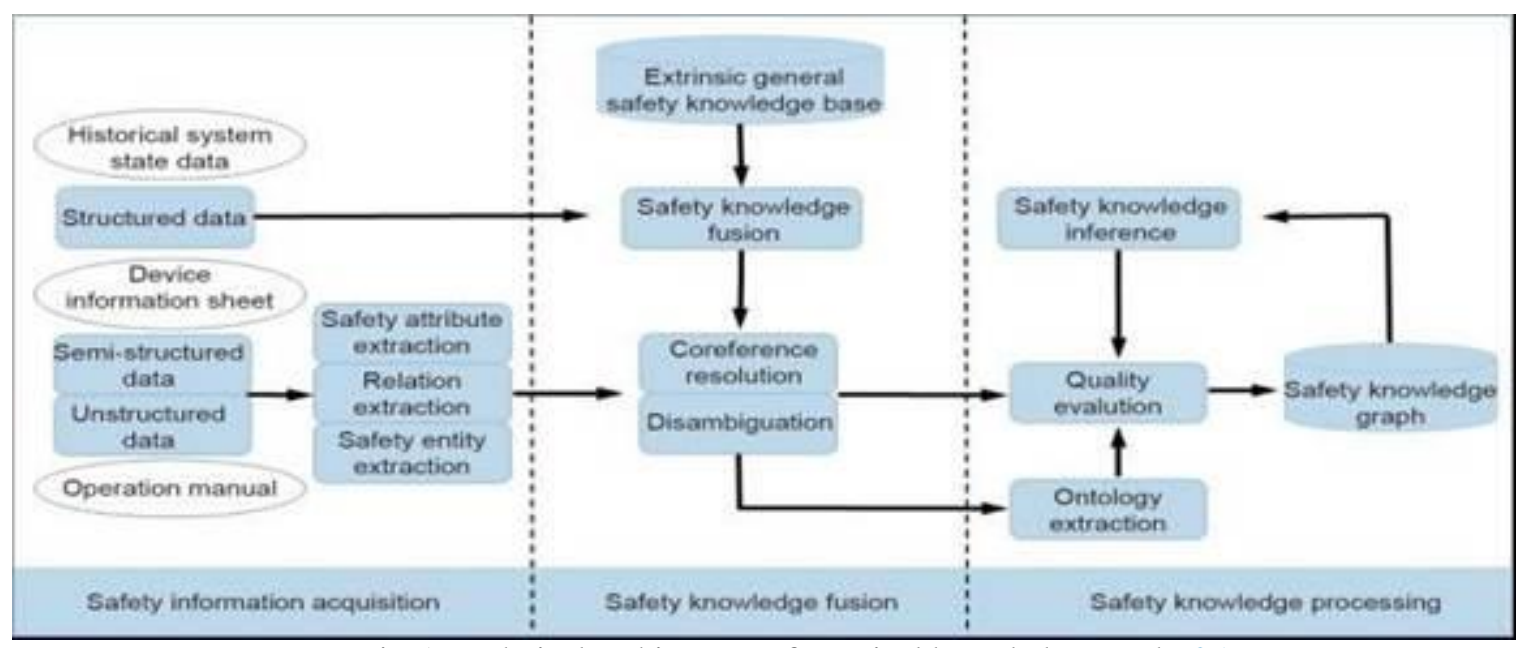

Fig.5 Technical architecture of a typical knowledge graph [25].

\subsection{Process-safety information extraction}

[26] The knowledge graph will be constructed with relevant information about certain factors like safety, chemicals, documents, controlling system, information, risk management factors, etc.

$[27,28]$ to collect the above-mentioned data diversity of information, coverage of information by the means of all aspects like tables, graphs, charts, texts, and diagrams, etc must be obtained. The main aim of the knowledge graph is to address linked data and to generate the information of individual entities and their relationship between information from various sources. This process of gathering information is known as the extraction of an entity, extraction of relationship, and extraction of an attribute.

Extraction of entity: This refers to the identification of entity from text automatically. It is the most beginning part of information extraction. In theprocess, factor information extraction depends on ontology schema inorder to obtain factors that cause risk. The data extraction is done through manual operation or through maintaining sheets, P \& IDs (piping and instrumentation diagrams), and PFD (process flow diagram)

Extraction of Relationship: It is the 2nd step in the process of extracting information in this step the relationship between the extracted entities will be identified. These steps will take place through semantic information or charts, tables, and diagrams. In the petrochemical industry, the information related to safety measures will be generated through documents. In order to know the relationship about factors causes risk will require a deep understanding of the factors reason for this kind of hazardous situation.

Extraction of Attribute: Each factor reason for accidents may have a different section of reasons to point out. In this step, all the attributes which are reasonable for creating an issue must be addressed and qualified to overcome the problem.

\subsection{Process-safety knowledge fusion}

[29] With the extraction of safety information the complete reason for risk involving factors and their relationship will be well-known. Anyone the obtained information may have errors and mistakes. The relationship obtained will be flat, improper connecting point, and logical. With the implementation of knowledge fusion, this kind of mistake or errors will be eliminated.

\subsection{Process-safety knowledge processing}

After the implementation of knowledge fusion, the errors will be eliminated. Then a set of factual articulations will be expressed. The analysis regarding safety is important in the process safety step. The reason for the main hazard and reason for the incidentmust be identified and avoided completely. Safetyrelated knowledge is needed for understanding the issue completely especially about the processing of chemicals, equipment, operation, and other mechanisms. The other smaller factors reason for issue must also be addressed gradually to identify the error in the beginning stage and the factor which is responsible for downstream must also be diagnosed with the help of predefined rules and knowledge. The knowledge 
processing involves 3 most important aspects which will be discussed in detail in the following section.

Safety ontology reconstruction: The term Ontology refers to a set of models and factors reason for describing the concept to the objective world. This term refers to the concept of knowledge and its relationship. This process can be developed through brainstorming with present knowledge.

Reason for risk in obtained relationship: [30,31] Reasoning helps in identifying the key relationship between the present entity based on a set of rules. Reasoning can be classified into reasoning based on logic-term, and reasoning based on the graphical method.

Assessment of quality: It is also one of the important aspects of developing a process safety knowledge graph. The obtained data will still have errors and mistakes even through obtained through the state-of-art method. Before the process of merging the newly obtained data and data obtained from the knowledge graph, a quality assessment isessential for evaluating the process. The knowledge graph provides a proper method for integrating information into the process industry. The problems like divergence in data, information gap, and representation of complex relationships will be addressed with thehelp of a knowledge graph.

\subsection{Risk assessment and decision-making using Bayesian networks}

[32,33] Bayesian networks are a type of Probabilistic Graphical Model that can be used to build models from data/ and expert opinion. In the processing factory, the reason for abnormalities may be due to several probabilistic relationships. The important 5 parameters must be addressed while analyzing hazardous reactions. Those 5 parameters include temperature, hot-material flow rate, heated material flow rate, and coking degree in furnace tubes. These mentioned parameters will have different probabilistic deviations.

The Bayesian network will address the complex probabilistic relationships between the abovementioned parameter and the runaway reaction. This process can be used for other kinds of accidents and other factors involving risk. In order to obtain accurate performance, the parameters of the Bayesian network must be handled carefully to achieve better performance. The Bayesian network can track the risk factors more accurately. And it can also provide a solution for an emergency situation depending on the abnormalities involved in it.

\subsubsection{Incident early warning based on deep learning}

[34]Deep learning (DL) is an artificial intelligence-based function that mimics the working of the human brain in processing data for the purpose of detecting objects, recognizing speech, language translation, and decision making. Which helps to learn without human intervention and supervision. In the process industry, the chances for accidents are mainly due to fluctuation in data. Composition change in the system may lead to more generation of heat which may narrow the working of the system. DL helpsin recognizing the reason for the accident and other factors involved in the occurrence of this accident will also be found. If clear information regarding data, equipment, and human operation was present in such a case the reason behind the accident will be found and steps to rectify it will be found.

[35] Labeling of big data is an important step in DL and other related techniques and identification of risk and its evaluation. But in reality, obtained big data may have mistakes and errors. So the present data are inadequate and with zero percent accuracy. Moreover, the industries will highly depend on expert advice and alarm from the system.

\section{Detailed Description of Common Encryption Algorithms}

The encryption algorithm helps in generating, modifying, and transporting key data. The encryption algorithm is also termed as a cryptographic algorithm. The power of the encryption algorithm is based on a Computer system which is utilized for generating keys. In this research paper, Triple-DES is used for encrypting data in order to maintain data confidentiality during the process of communication.

\section{Triple DES (3DES) - (Triple Data Encryption Standard)}

The 3DES may also be referred to us as TDEA (Triple Data Encryption Algorithm) which was developed by IBM during 1998. This was found to be a replacement of DES because of the improved structure 
and DES was applied 3 times in the data block. The DES with 56 bit was enough in the initial time during the early designing process but in a later period due to an increase in power of computing the attack realizable.

3DES is a simplistic method while considering the design pattern of the whole block cipher and its capacity against forceful attack. The forceful attack tries to attack harder until getting the original message. Table 1 illustrates the 4 key sizes. The DES is embedded with a 56-bit key size and 3DES with 138 bit. The length of the key for 3DES is 112 and 168 bits respectively for 48 rounds and the size of the block is 64 . The main aim of this algorithm is to maximize security with a longer length key. The Ks- size of the key, Nknumber of keys, Tr 1 D- time needed for one decryption, and Tr 106 D- timeneeded for 106 decryption.

The advantage of 3DES is considered as thrice the size of the key (2168) when compared to the key size (256) of DES. This is the main reason for 3DES to be preferred

over DES. It gives an adequate amount of security for the information but at the sametime, it requires more time for encryption when compared to DES.

The 3DES algorithm is represented as follows:

$$
\begin{aligned}
& \quad \mathrm{C}= \\
& \text { EncryptK3(DecryptK2(EncryptK1( } \\
& \text { P) }
\end{aligned}
$$

(3) and decryption algorithm of 3DES is given as follows:

$\mathrm{P}=$ DecryptK3(EncryptK2(DecryptK1(C)

(4) Where $\mathrm{C}$ represented the ciphertext, $\mathrm{P}$ represented the plaintext and $\mathrm{K} 1, \mathrm{~K} 2$, K3represent the keys.

(5) The 3DES in the following years shall be defined as

- To overcome the forceful attack of the DES with the utilization of 168 bits size of the key.

- The 3DES is almost similar to that of the DES in many ways.

The 3DES algorithm for the purpose of security will be the best choice for a standard encryption algorithm in the future. The main disadvantage of 3DES was considered to be the slower set of software systems because it was developed in the early 1970s and the software is not efficient for the present time. The reason for the slow software structure isbecause it involves 3 more additional rounds.

The 2nd drawback in DES and 3DES is that the size of the block is 64 bit which is used and in the aspect of security the larger block size is preferred.

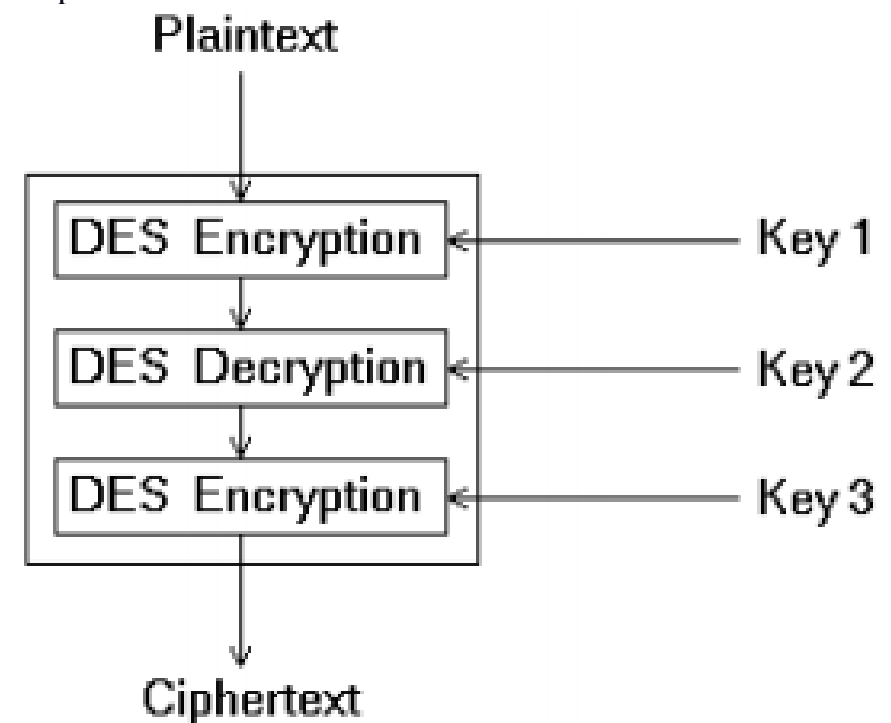

Fig. 6 3DES Structure

\section{Current and Future Works}

To understand Green Manufacturing through Artificial Intelligence studies have been initiated relating to processing safety knowledge in delaying the coking process. The outcomes of this present paper are illustrated in fig 7. The fig describes the entire method involved in this present research. In the aspect of the knowledge graph, all the elements related to safety are observed including all the upper and lower factors involved in the parameter, the up and downstream in a relationship the reason for another parameter for the cause of the accident was also considered.

The visualization process and question answering was conducted to enhance the interaction 
between machine and human, which is an important factor for SM (Smart Manufacturing)

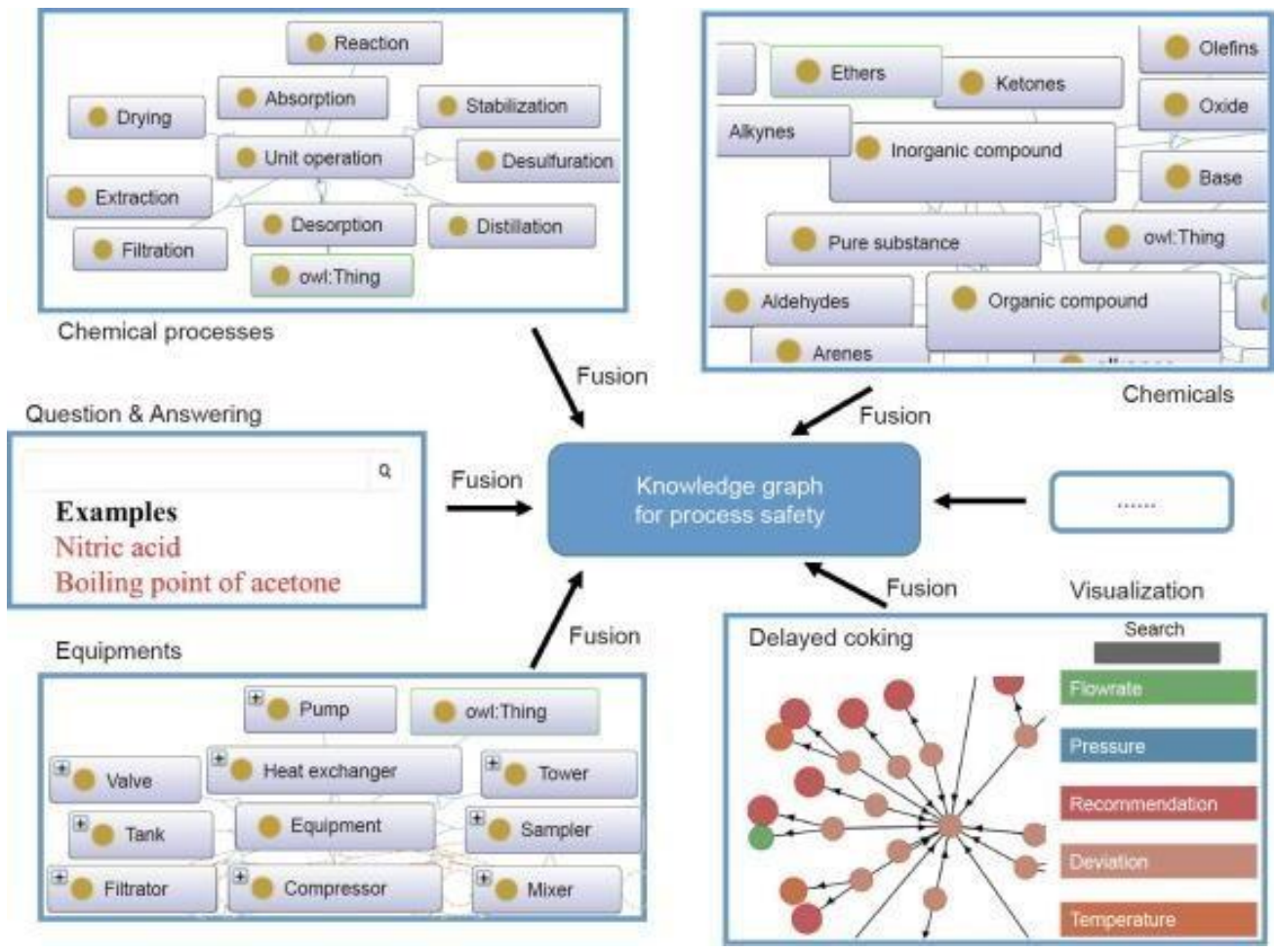

Fig. 7. Framework of current and future works.

The cause and effect relationship between arbitrary deviation and their involvement downstream will be given more importance in the future study. The downstream at the initial level will be analyzed with the help of decision making. The main aim is to address the current abnormal situation through the process monitoring method and to permit logical analysis to address the simple fault and events automatically. With the involvement of more detailed data the knowledge graph, tracking of risk, and the process of decision making will be attained in future research proposals with the help of deep learning (DL) and Bayesian network.

\section{Conclusions}

This present research paper addressed the significance, present status, and other problems involved in Green Manufacturing in the terms of the process industry. Various techniques with the usage of Artificial Intelligence have been reviewed which includes the Bayesian network, knowledge graph, and DL. These factors helped in solving a significant problem faced by Green Manufacturing. The technical challenges in process safety were also discussed. Some of the challenges involved are the acquisition of knowledge and reasoning regarding error and mistakes in obtained data, beforehand warning regarding error/accident, and effective decision making. The possible factors for addressing the above-mentioned challenges were proposed and other achievements were detailed.

\section{References}

Mao, Shuai, et al. "Opportunities and challenges of artificial intelligence for green manufacturing in the process industry." Engineering 5.6 (2019): 995-1002.

F. Qian, W. Zhong, W. DuFundamental theories and key technologies for smart and optimal manufacturing in the process industry Engineering, 3 (2) (2017), pp. 154-160

C.A. Giffi, M.D. Rodriguez, B. Gangula, A.V. Roth, T. Hanley Globalmanufacturing competitiveness index Deloitte Touche Tohmatsu Limited Global Consumer \& Industrial Products Industry Group and the Council on Competitiveness, London (2016)

E. WilliamsEnvironmental effects of information and communications technologies Nature, 479 (7373) (2011), pp. 354-358.

Smart Manufacturing Leadership Coalition. Implementing 21st century smart manufacturing: workshop summary report. Washington: Smart Manufacturing Leadership Coalition; 2011.

State Council of the People's Republic of China. [New generation of artificial intelligence development plan] 
[Internet]. Beijing: State Council of the People's Republic of China; 2017 Jul 8 [cited 2019 May 8].

Z. Yuan, W. Qin, J. ZhaoSmart manufacturing for the oil refining and petrochemical industry Engineering, 3 (2) (2017), pp. 179-182.

J. Zhou, P. Li, Y. Zhou, B. Wang, J. Zang, L. MengToward new-generation intelligent manufacturing Engineering, 4 (1) (2018), pp. 11-20

R. CernanskyChemistry: green refill Nature, 519 (7543) (2015), pp. 379-380

S.J. Russell, P. NorvigArtificial intelligence: a modern approach Pearson Education Limited, Kuala Lumpur (2016)

D. Silver, A. Huang, C.J. Maddison, A. Guez, L. Sifre, G. van den Driessche, et al.Mastering the game of Go with deep neural networks and tree searchNature, 529 (7587) (2016), pp. 484-489

I.D.L. BogleA perspective on smart process manufacturing research challenges for process systems engineers Engineering, 3 (2) (2017), pp. 161-165.

T. ChaiIndustrial process control systems: research status and development direction Sci Sin Inf, 46 (8) (2016), pp. 1003-1015 Chinese

S.M. Tauseef, T. Abbasi, S.A. AbbasiDevelopment of a new chemical process- industry accident database to assist in past accident analysis J Loss Prev Process Ind, 24 (4) (2011), pp. 426-431

P. Huang, J. ZhangFacts related to August 12, 2015 explosion accident in Tianjin, China Process SafProg, 34 (4) (2015), pp. 313-314

B. Wang, C. Wu, G. Reniers, L. Huang, L. Kang, L. ZhangThe future of hazardous chemical safety in China: opportunities, problems, challenges and tasks Sci Total Environ, 643 (2018), pp. 1-11

J. BondProfessional ethics and corporate social responsibility Process Saf Environ Prot, 87 (3) (2009), pp. 184190

D.A. Dornfeld

Green

manufacturing:

fundamentals

and applications, Springer, New York (2013)

J.H. ClarkGreen chemistry: challenges and opportunities Green Chem, 1 (1) (1999), pp. 1-8

BASF Corporation. Fire at the North Harbor in Ludwigshafen [Internet]. Ludwigshafen: BASF Corporation; 2016 Oct 27 [cited 2019 May 8].

BASF Corporation. German firefighter dies 11 months after BASF explosion [Internet]. Haarlem: Expatica; 2017 Sep 5 [cited 2019 May 8].

Z. Qu, H. Feng, Z. Zeng, J. Zhuge, S. JinA SVM-based pipeline leakage detection and pre-warning system Measurement, 43 (4) (2010), pp. 513-519

H. PaulheimKnowledge graph refinement: a survey of approaches and evaluation methods Semant Web, 8 (3) (2017), pp. 489-508

M. Färber, F. Bartscherer, C. Menne, A. RettingerLinked data quality of DBpedia, Freebase, OpenCyc, Wikidata, and YAGO Semant Web, 9 (1) (2018), pp. 77-129

Ehrlinger L, Wöß W. Towards a definition of knowledge graphs. In: Proceedings of SEMANTICS 2016: posters and demos track; 2016 Sep 13-14; Leipzig, Germany; 2016.

Q. Liu, Y. Li, H. Duan, Y. Liu, Z. QinKnowledge graph constructiontechniques J Comput Res Dev, 53 (3) (2016), pp. 582-600.

S.E. Gordon, K.A. Schmierer, R.T. GillConceptual graph analysis: knowledge acquisition for instructional system design Hum Factors, 35 (3) (1993), pp. 459-481.

Miwa M, Sasaki Y. Modeling joint entity and relation extraction with table representation. In: Proceedings of the 2014 Conference on Empirical Methods in Natural Language Processing; 2014 Oct 25-29; Doha, Qatar; 2014.p. 1858-69.

G. Paliouras, C.D. Spyropoulos, G. TsatsaronisKnowledge-driven multimedia information extraction and ontology evolution: bridging the semantic gap Springer, Heidelberg (2011).

X.L. Dong, E. Gabrilovich, G. Heitz, W. Horn, K. Murphy, S. Sun, et al.From data fusion to knowledge fusion Proc VLDB Endowment, 7 (10) (2014), pp. 881-892.

X. Wang, T. Gu, D. Zhang, H.K. PungOntology based context modeling and reasoning using OWL Proceedings of the 2nd IEEE Annual Conference on Pervasive Computing and Communications Workshops; 2004 Mar 14-17;Washington, DC, USA, IEEE, New York (2004), pp. 18-22.

B. Kamsu-Foguem, D. NoyesGraph-based reasoning in collaborative knowledge management for industrial maintenanceComputInd, 64 (8) (2013), pp. 998-1013.

J. Zhu, Z. Ge, Z. Song, L. Zhou, G. ChenLarge-scale plant-wide process modeling and hierarchical monitoring: a distributed Bayesian network approach J Process Contr, 65 (2018), pp. 91-106.

P. Larrañaga, H. Karshenas, C. Bielza, R. SantanaA review on evolutionaryalgorithms in Bayesian network learning and inference tasks InfSci, 233 (2013), pp. 109-125.

Y. LeCun, Y. Bengio, G. HintonDeep learning Nature, 521 (7553) (2015),pp. 436-444.

J. Zhu, Z. Ge, Z. Song, F. GaoReview and big data perspectives on robust data mining approaches for industrial process modeling with outliers and missing data Annu Rev Contr, 46 (2018), pp. 107-133. 
Z. Zhou, G. Qi, B. GlimmExploring parallel tractability of ontology materialization Proceedings of the 22nd European Conference on Artificial Intelligence; 2016 Aug 29-Sep 2; Amsterdam, the Netherlands, IOS Press, Amsterdam (2016), pp. 73-81. 